$\mathbb{P}$ periodica polytechnica

Mechanical Engineering

$55 / 1(2011) 21,27$

doi: 10.3311/pp.me.2011-1.03

web: http://www.pp.bme.hu/me

(c) Periodica Polytechnica 2011

RESEARCH ARTICLE

\section{Syntactic foams produced by pressure infiltration - the effect of pressure and time on infiltration length}

\author{
Imre Norbert Orbulov
}

Received 2011-04-30

\begin{abstract}
Ceramic hollow microballoons were incorporated into aluminium matrix to produce metal matrix syntactic foams (MMSFs). Syntactic foams have low density and relatively high strength; therefore they can be used as materials of many important parts in automotive technology or in aviation and sea industry. The MMSFs can be produced by pressure infiltration technique, which can be productive if it is modified in the direction of injection casting. The aim of this paper is to determine the necessary pressure and time parameters for the successful infiltration of a given length. A measurement system was developed and applied to investigate the effect of pressure and time on infiltrated length. In this paper the results of these preliminary measurements are presented. Power law ruled surface was fitted on the measured points in order to evaluate the infiltrated length for any pressure and time parameters. The results were compared to the theoretically predicted ones and on this base an advanced measurement system is suggested and designed.
\end{abstract}

\section{Keywords}

syntactic foam - metallic foam - metal matrix composite $\cdot$ pressure infiltration · ceramic microballoons $\cdot$ infiltration parameters

\section{Imre Norbert Orbulov}

Department of Materials Science and Engineering, BME, Bertalan Lajos street 7. H-1111, Budapest,, Hungary

e-mail: orbulov@eik.bme.hu

\section{Introduction}

Metal matrix syntactic foams (MMSFs) are porous materials but they can also be classified as particle reinforced composites. The porosity is ensured by introducing ceramic hollow spheres (microballoons) into the matrix material, which is normally a kind of aluminium alloy. The volume fraction of the microballoons normally varies between 30 and 70 vol\% and they contain mainly $\mathrm{Al}_{2} \mathrm{O}_{3}(35-40 \%)$ and $\mathrm{SiO}_{2}(55-60 \%)$. The MMSFs are good energy absorbers and mechanical dampers. They have low weight, outstanding specific properties, localized failure, good creep resistance, etc. The specific mechanical properties of MMSFs can be 50-100\% higher than the "conventional"foams [1,2]. They are used as energy absorbers and sound absorbers or as material of hulls and shells in deep-sea applications and in aeronautics or avionics.

MMSFs can be made by pressure infiltration or blending method. Pressure infiltration is the most common and the most promising way to produce MMSFs, because that is the closest method to the widely spread die-casting [3]. Rohatgi et al used pressure infiltration to infiltrate fly ash particles and found that the strength of the composite increases with an increase in the volume fraction of the particles [4,5]. Palmer et al. used Al1350, Al5083 and Al6061 aluminium as matrix material. The different syntactic foams were characterized and compared by upsetting, tensile and bending tests [6]. Balch et al. also reported successful production of syntactic foams by pressure infiltration and they studied load partitioning and load transferring effects between the microballoons and the matrix [7, 8].

In order to have successful infiltration a threshold pressure must be assured by the infiltrating system. This threshold pressure can be calculated by theoretical and experimental approaches for various systems. When the required pressure is below 1 bar the pressure difference can be generated by vacuum instead of inert gas pressure. Kiser et al. investigated the compressive behaviour of syntactic foams produced by vacuum assisted casting. They also predicted the compressive strength of the foams in cases of different stress fields [9]. Nowadays, the mathematical description of syntactic foams is important in order to perform correct economic design; due to this $\mathrm{Wu}$ et 
al. gave a prediction of the compressive strength of MMSFs, showing the relation among microballoon diameter wall thickness and the strength [10]. Kaptay et al. developed a fully theoretical model for closely packed spheres (CPES) to predict the threshold pressure for infiltration. The model based on the equilibrium of gravitational, capillary and outer forces and considered the effect of wetting angle, surface tension and volume fraction [11].

$$
\begin{aligned}
& \frac{p R}{\sigma_{1 / g}}=\frac{\sqrt{3}}{3} \frac{\pi}{\varepsilon_{S}}\left(\frac{h}{R}-1-\cos \theta\right)+ \\
& \frac{\sqrt{3}}{3} \frac{\pi}{\varepsilon_{S}}\left(\frac{h}{R}-2.63-\cos \theta\right)
\end{aligned}
$$

in which

$$
\begin{aligned}
& \varepsilon_{s}=1-A\left|2 \frac{h}{R}-\left(\frac{h}{R}\right)^{2}\right| \\
& -A\left|2\left(\frac{h}{R}-1.63\right)-\left(\frac{h}{R}-1.63\right)^{2}\right|
\end{aligned}
$$

where $p$ is the pressure, $R$ is the average diameter of the microballoons, $\sigma_{l / g}$ is the interfacial energy between the infiltrating liquid (the melt) and the surrounding gas (air), $\varepsilon_{s}$ is the ratio of the liquid/gas interface and the total cross-section area, $\mathrm{A}$ is a coefficient for the volume fraction of the microballoons ( $A=0,906$ for CPES modell), $h$ is the vertical position of the infiltrating melt front, $\theta$ is the wetting angle between the material of the microballoons and the infiltrating material. We modified this model into the case of "randomly close packed equal spheres" (R-CPES), which can be achieved by pouring the microballoons in a container and ordering them by tapping. It was proved experimentally; the volume fraction of the microballoons converges logarithmically to $64 \mathrm{vol} \%$ (instead of $74 \mathrm{vol} \%$ for CPES modell) according to the number of tapping [12]. In this case, by assuming equal distance between the microballoons the coefficient A became 0,822 . Trumble defined a hydraulical radius, which depended on the shape and the volume fraction of the fillers and calculated the threshold pressure by using the Young-Laplace equation [13].

$$
p=\frac{2 \sigma_{1 / g} \cos \theta}{r_{h}}=\frac{12 \lambda\left(1-V_{m b}\right) \sigma_{1 / g} \cos \theta}{V_{m b} 2 R}
$$

where all designation is the same as in the Kaptay equation but $r_{h}$ is the hydraulical radius, $V_{m b}$ is the volume fraction of the microballoons (64 vol\%) and $\lambda$ is a shape factor an equals to 1 in case of spheres. The same was done by Rohatgi et al., but they defined an effective distance between the microballoons. The base equation was the Young-Laplace equation again [5].

$$
p=\frac{2 \sigma_{1 / g} \cos \theta}{r_{e}}=\frac{2 \sigma_{1 / g} \cos \theta V_{m b}}{2 R\left(1-V_{m b}\right)}
$$

where every designation in the same and $r_{e}$ stands for the effective distance. We calculated the required threshold pressures by the interpreted models with typical parameters: the average diameter of the microballoons was considered to $130 \mu \mathrm{m}$. The interfacial energy was $860 \times 10^{-3} \mathrm{Nm}^{-1}$, this corresponds to oxidized surface for aluminium alloys. The wetting angle was varied between $0^{\circ}$ and $180^{\circ}$ to represent the varying of the threshold pressure. The calculated threshold pressures are shown in Fig. 1.

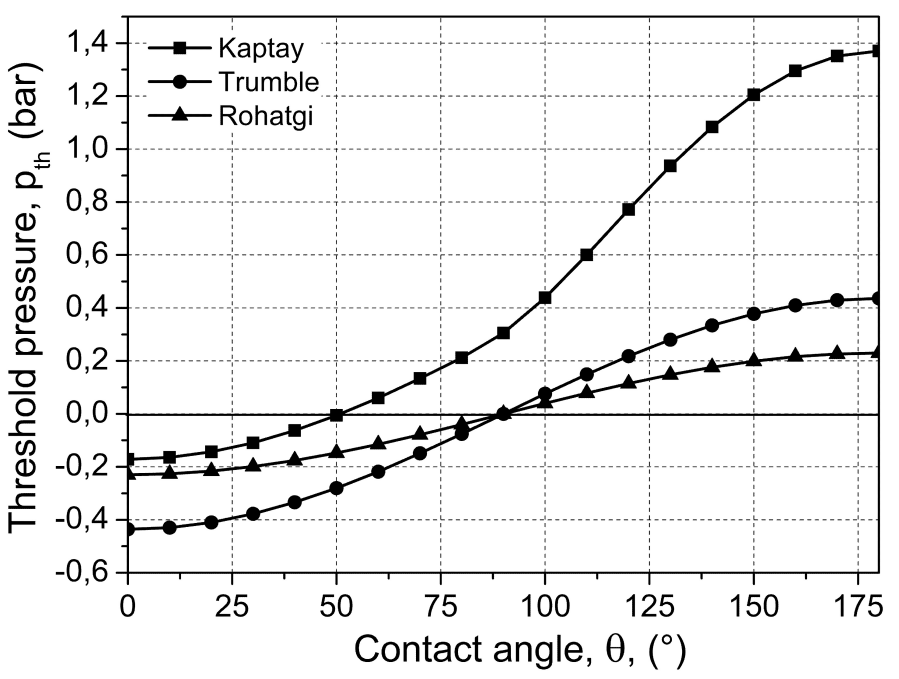

Fig. 1. The treshold pressure as the function of wetting angle

The Trumble and Rohatgi model has the same critical contact angle $\left(90^{\circ}\right.$, the border between wetting and non-wetting states); however the Kaptay model showed that the real critical contact angle is about $50^{\circ}$ due to capillary effects. The contact angle between the material of the microballoons (compound of $\mathrm{SiO}_{2}$ and $\mathrm{Al}_{2} \mathrm{O}_{3}$ ) and the aluminium alloys is between 140 and $160^{\circ}$. In this range the models developed by Rohatgi et al. and by Trumble predict significantly smaller threshold pressures than the Kaptay modell. Therefore measurements are required to verify the theoretically predicted values. Moreover these equations give threshold pressure only and they do not consider the effect of higher pressure levels and longer time, which are technological parameters and have direct effect on the infiltrated length. To clear these problems we developed a measurement method to demonstrate the effect of these parameters.

\section{Materials and experimental methods}

AlSi12 aluminium alloy was used as matrix material. This alloy has low melting point, very good castability and fluidity; therefore it is ideal for pressure infiltration. As microballoons, SLG type of E-spheres were used (supplier: Envirospheres Pty. Ltd. [14]). E-Spheres have low real density $\left(\sim 640 \mathrm{kgm}^{-3}\right)$ and favourable chemical composition (36-40 wt $\% \mathrm{Al}_{2} \mathrm{O}_{3}, 55-60$ wt $\% \mathrm{SiO}_{2}, 1.4-1.6 \mathrm{wt} \% \mathrm{TiO}_{2}$ and 0.4-0.5 wt $\% \mathrm{Fe}_{2} \mathrm{O}_{3}$ ). Their average outer diameter was $130 \mu \mathrm{m}$ and they had average wall thickness $\sim 5.4 \mu \mathrm{m}$ (calculated from the real density of the microballons and from the density of their wall). Inert Ar gas was used as a pressurizing agent. To investigate the effect of infiltration pressure and time we have developed an equipment shown in Fig. 2 The whole system was made from stainless steel (316L). 
$\begin{array}{llllllllllllll}1 & 2 & \underline{3} & 11 & 10 & \underline{9} & \underline{8} & \underline{7} & \underline{6} & \underline{5} & \underline{4} & \underline{3} & \underline{2} & 1\end{array}$

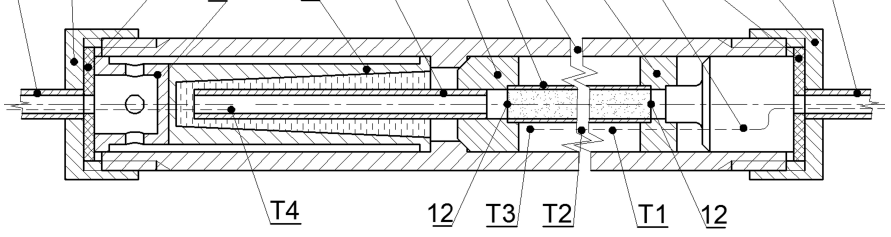

Fig. 2. Experimental infiltration equipment $\left(90^{\circ}\right.$ rotated view $)$

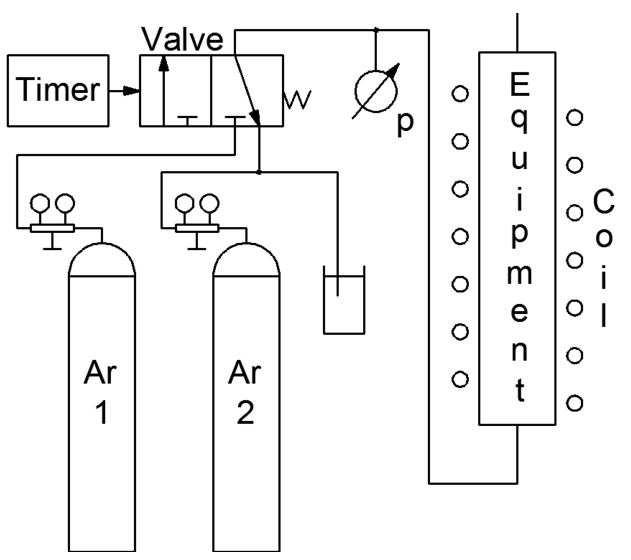

Fig. 3. Arrangement of the measurements

The main part of the equipment is the inner tube (\#7), which has $10 \mathrm{~mm}$ outer diameter, $150 \mathrm{~mm}$ in length and has $1 \mathrm{~mm}$ wall thickness. The tube was fulfilled with microballons and they were tapped rigorously in order to achieve $\sim 64 \%$ volume fraction. Then a previously machined matrix material ingot was placed in the crucible (\#10). After that thermocouples (marked by letter "T1...4" in the picture) were placed in and the whole system was assembled. Three thermocouples (T1...3) monitored the temperature of the inner tube and one measured the temperature of the matrix material (T4). The inner elements were fixed in their places by spacers (parts \#4, 5 and 11). The whole testing procedure was thermally controlled. Between the cups (\#2) and the main tube (\#6) insulator layers were placed (\#3). Part \#8 divides the main tube (\#6) into two chambers. The chambers are connected to the environment or to the other parts of the system through pipes (\#1). At the beginning of the procedure both chambers were flushed by Ar gas. The arrangement of the measurements is shown in Fig. 3 .

At the beginning of the procedure the electrically operated valve was in the position shown in the picture. The Ar gas, from vessel designated Ar2, was flushing through the equipment with minimal pressure equals to about $20 \mathrm{~mm}$ of water height). After a few minutes we began heating the equipment with induction coil (Power-Trak 15-96). Temperature changes were registered by the previously placed thermocouples. During melting $\mathrm{Ar}$ flushing was continuous and the pressure of another vessel (Ar1) was set to the required infiltration pressure. When the matrix alloy melted and superheated (melting point $+70^{\circ} \mathrm{C}$ ), the valve was operated through a timer. The timer switched the valve and therefore infiltration pressure appeared at the lower chamber of the equipment. The upper chamber remained opened to the en- vironment, so a pressure difference could develop between the upper and lower chambers. This pressure difference forced the matrix material infiltrate the microballoons through the tube (\#9) and could be measured by a built-in pressure gage. When the previously set time ran down the valve was switched back by a spring. Then the equipment was removed from the induction coil and cooled down in water. The inner tube was removed and the infiltrated length was measured. Each test was done with different pressure and time parameters to find out their effects on the infiltrated length.

\section{Results and discussion}

The time, pressure and temperature parameters of the experiments and the resulted infiltrated lengths are shown in Tab. 1 .

The duration of pressure infiltrations were 1,3 or 5 seconds, while pressure varied between 1 and 4 bars. The pressure increment was 1 bar, so 12 different parameter pairs were tested. Some measurements were repeated in order to investigate reproducibility. These measurements were marked by asterisk and the values in those rows are the average of the original and repeated measurements. $T 1, T 2$, and $T 3$ show the temperatures in the inner tube. $T 1$ was at the top of the tube, $T 2$ was in the middle and $T 3$ was at the bottom. T4 measured the temperature of the matrix material. The first row shows that below 0.5 bar there was no infiltration, therefore the real threshold pressure for the investigated system is between 0.5 and 1 bar. This validates the Kaptay model in such a manner that the model predicts higher threshold pressure in the $140-160^{\circ}$ contact angle range. The infiltrated lengths were plotted above pressure - time plane as shown in Fig. 4.

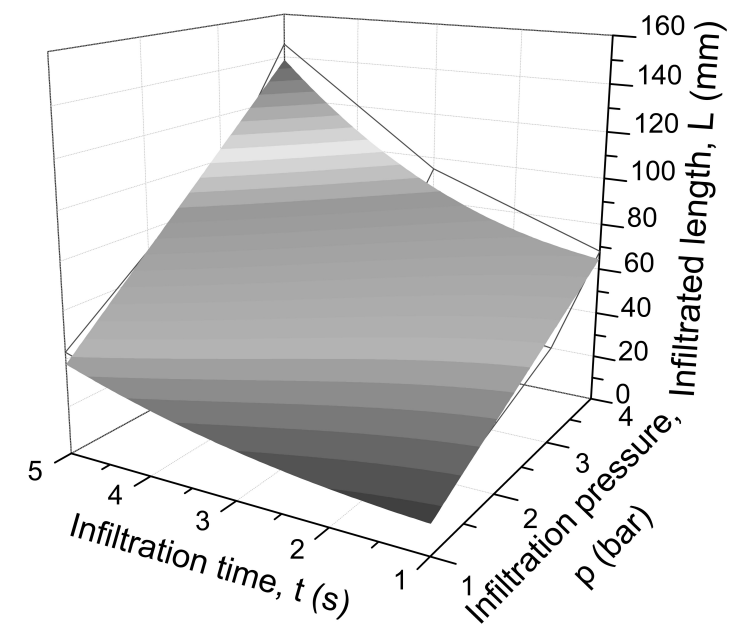

Fig. 4. The infiltrated lengths above the p-t plane with the fitted surface

This graph shows the increase of infiltrated length as the infiltrating pressure or time is increased. This means that a given infiltrated length can be achieved by increasing the infiltrating pressure or time. In the industry higher pressure requires extra costs due to more complicated seals and more precious machining.

On the other hand increasing infiltrating time can result in the 
Tab. 1. Parameters of the experiments and the infiltrated lengths

\begin{tabular}{|c|c|c|c|c|c|c|c|}
\hline \multirow{2}{*}{$\begin{array}{l}\text { Number of } \\
\text { experiment }\end{array}$} & \multirow[t]{2}{*}{ Time,t(s) } & \multirow{2}{*}{$\begin{array}{l}\text { Pressure, } \\
\mathrm{p} \text { (bar) }\end{array}$} & \multirow{2}{*}{$\begin{array}{l}\text { Infiltrated length, } \\
\mathrm{L}(\mathrm{mm})\end{array}$} & \multicolumn{4}{|c|}{ Temperature $\left({ }^{\circ} \mathrm{C}\right)$} \\
\hline & & & & $\begin{array}{l}\mathrm{T} 1 \\
\left({ }^{\circ} \mathrm{C}\right)\end{array}$ & $\begin{array}{l}\mathrm{T} 2 \\
\left({ }^{\circ} \mathrm{C}\right)\end{array}$ & $\begin{array}{l}\mathrm{T} 3 \\
\left({ }^{\circ} \mathrm{C}\right)\end{array}$ & $\begin{array}{l}\mathrm{T} 4 \\
\left({ }^{\circ} \mathrm{C}\right)\end{array}$ \\
\hline 0. & 5 & 0.5 & 0 & 660 & 650 & 630 & 640 \\
\hline $1 .^{*}$ & 1 & 1 & 17 & 640 & 640 & 620 & 640 \\
\hline 2. & 1 & 2 & 29 & 640 & 630 & 620 & 640 \\
\hline 3. & 1 & 3 & 41 & 640 & 640 & 620 & 640 \\
\hline 4. & 1 & 4 & 68 & 610 & 630 & 630 & 640 \\
\hline 5. & 3 & 1 & 21 & 670 & 670 & 640 & 640 \\
\hline 6. & 3 & 2 & 41 & 640 & 640 & 625 & 640 \\
\hline 7.* & 3 & 3 & 54 & 680 & 670 & 645 & 640 \\
\hline 8. & 3 & 4 & 95 & 650 & 650 & 630 & 640 \\
\hline 9. & 5 & 1 & 43 & 710 & 710 & 690 & 640 \\
\hline 10. & 5 & 2 & 71 & 700 & 690 & 700 & 640 \\
\hline 11. & 5 & 3 & 94 & 670 & 660 & 665 & 640 \\
\hline $12 .^{*}$ & 5 & 4 & 146 & 670 & 640 & 630 & 640 \\
\hline Average & & & & 660 & 655 & 642 & 640 \\
\hline Scatter & & & & 27.4 & 24.0 & 26.7 & 0 \\
\hline
\end{tabular}

Tab. 2. Parameters of the power surface

\begin{tabular}{llllll}
\hline $\mathrm{L}_{0}(\mathrm{~mm})$ & $\mathrm{C}_{1}\left(\mathrm{~mm} \times \mathrm{bar}^{-1}\right)$ & $\mathrm{C}_{2}\left(\mathrm{~mm} \times \mathrm{s}^{-1}\right)$ & $\mathrm{C}_{3}(-)$ & $\mathrm{C}_{4}\left(\mathrm{~mm} \times \mathrm{bar}^{-1} \times \mathrm{s}^{-1}\right)$ & $\mathrm{R}^{2}(-)$ \\
\hline-5.37214 & 16.95518 & 0.26065 & 2.25129 & 0.44382 & 0.95334 \\
\hline
\end{tabular}

Tab. 3. Fitting parameters of the power equation, the time is constant

Tab. 4. Fitting parameters of the fitted power curves, the pressure is constant

\begin{tabular}{|c|c|c|c|c|c|}
\hline & $\begin{array}{l}\text { Infiltration } \\
\text { time, } \\
t(s)\end{array}$ & \multicolumn{3}{|c|}{ Fitting parameters } & \\
\hline & & \multicolumn{2}{|l|}{$\mathrm{L}_{p 0}(\mathrm{~mm})$} & $\mathrm{C}_{p}\left(\mathrm{~mm} \times \mathrm{bar}^{-1}\right)$ & \\
\hline & 1 & \multicolumn{2}{|l|}{-2.5} & 16.5 & \\
\hline & 3 & \multicolumn{2}{|l|}{-6} & 23.5 & \\
\hline & 5 & 5.5 & \multicolumn{2}{|c|}{33.2} & \\
\hline \multirow{2}{*}{\multicolumn{2}{|c|}{$\begin{array}{l}\text { Infiltration } \\
\text { sure, } \\
p \text { (bar) }\end{array}$}} & \multicolumn{3}{|c|}{ Fitting parameters } & \\
\hline & & $\begin{array}{l}\mathrm{L}_{t 0} \\
(\mathrm{~mm})\end{array}$ & $\begin{array}{l}\mathrm{C}_{t 1} \\
(\mathrm{~mm} \times \\
\left.\mathrm{s}^{-1}\right)\end{array}$ & $\mathrm{C}_{t 2}(-)$ & $\mathrm{t}_{p}(\mathrm{~s})$ \\
\hline 1 & & 17.17 & 0.61538 & 2.70044 & 1 \\
\hline 2 & & 29.29 & 3.42857 & 1.80735 & 1 \\
\hline 3 & & 41.41 & 3.18868 & 2.02748 & 1 \\
\hline 4 & & 68.68 & 9.34615 & 1.53051 & 1 \\
\hline
\end{tabular}

development of chemical reactions (when it is possible between the matrix and microballoons, in our case for example:

$$
\left.4 \mathrm{Al}+3 \mathrm{SiO}_{2}=3 \mathrm{Si}+2 \mathrm{Al}_{2} \mathrm{O}_{3}, \Delta \mathrm{G} \approx-300 \mathrm{~kJ} / \mathrm{mol} \text { at } 700-850^{\circ} \mathrm{C}\right) .
$$

Therefore an optimum should be searched between the parameters. The graph is very useful, if the parameters can be determined. But in that case some mathematical formulae are needed. A possible way is the linear interpolation between the measured points. This is the simplest method, but it is not too precious. A much better way is to fit a non-linear surface on the measured points. In that case the equation of the surface will be given in analytical form and the length can be determined for any parameters. The author tried to fit a few functions. A sufficient fit was ensured by an equation, showing linear behaviour with pressure and exponential behaviour with time. The equation uses five parameters as detailed in the followings.

$$
L(p, t)=L_{0}+C_{1} p+C_{2} t^{C 3}+C_{4} p t^{C 3}
$$

The parameters were determined by fitting and listed in Tab. 2 . The quality of the fitting can be described by its $R^{2}$ value, which 
is near to 1 and therefore the fitting is good enough.

With the equation of the surface the infiltrated length can be easily determined within the measured ranges and in a reverse case, when a desired infiltration length is given, one can evaluate satisfying infiltration pressure and time parameters. To investigate the effect of time and pressure independently we made sections along constant pressure and time values. After that linear and non-linear curves were fitted on the measured points. Again power curves were used and compared to the sections made from the fitted surfaces. The measured points, fitted curves and the sections of the surfaces are plotted in Fig. 5

The equations of the fitted curves were the followings. For a given and constant infiltration time a linear equation should be fitted on the points, which means that the infiltrated length is linear function of the infiltrating pressure.

$$
L_{p}=L p_{0}+C_{p} p
$$

For a given and pressure is constant a power ruled equation gives the best fitting.

$$
L_{t}=L_{t 0}+C_{t 1}\left|t-t_{p}\right|^{C_{t 2}}
$$

The power equation uses four parameters, which were determined by fitting and listed in Tab. 3 and Tab. 4.

Fig. 5 a shows that, the infiltrated length changes proportional to the pressure. As the pressure drops to zero the infiltrated length should be zero also in non-wetting conditions. The interception with the length axes is shown by $\mathrm{L}_{p 0}$ parameter. In Tab. 3 one can observe that this parameter is near to zero (within the scattering of the measurements). In the case of $t=5 \mathrm{~s}$ the $\mathrm{L}_{p 0}$ constant became positive, which means that after $5 \mathrm{~s}$ contact the system may become wetting (due to chemical reactions for example) and $5.5 \mathrm{~mm}$ would be infiltrated spontaneously at zero pressure. In the case of $t=1$ and $3 \mathrm{~s}$ a given $p>0$ threshold pressure is required to achieve some infiltration (non-wetting conditions). These pressures were almost the same in both cases and should be similar in the third case $(t=5 s)$. However when $t=5$ seconds a negative threshold pressure could be calculated, the infiltration could be prevented by this pressure (wetting conditions). This means that in the case of $t=5$ the wetting conditions were modified by something, maybe the possible chemical reaction between the constituents and the system became wetting. Fig. 5b and the parameters in Tab. 4 show that the infiltration length increased with exponent between $\sim 1.5$ and 2.7. These results are not in agreement with the previously published ones, because they predict that the infiltrated length should be proportional to the square root of time (the exponent should be $0.5)$. However these theoretical works neglected many physical parameters in order to simplify the calculations. For example:

- geometrical simplifications of real problems are made in order to get equations can be solved by analytical methods (regular spatial distribution of capillaries instead of random distribution, connectivity and tortuosity of the capillaries, etc.).
- the change in the permeability of the infiltrated preforms is neglected.

- the chemical reactions between the reinforcement and matrix is neglected (the reaction rate can be high at temperatures above liquidus and generally reduces the surface tension).

- the time dependence of the wetting angle is neglected.

- the thixotropic dilation and rupture of the oxide membrane on the infiltration front are neglected.

- the air resistance is neglected.

- the gravitational force is neglected.

Washburn studied the penetration of liquids into cylindrical capillaries and porous bodies in 1921 and found that, the infiltrated length should change proportional to the square root of time. However Washburn considered the shortcomings of the simplified theoretical equations and stated that in a given case the infiltrated length could only be determined by measurements [15]. Semlak and Rhines modelled porous metal bodies in 1958. They assumed that a powder compact consists of a great many parallel capillary tubes. Their results showed that the infiltration length is a linear function of the square root of time and influenced by the diameter of the capillaries, the wetting angle, the viscosity and the surface tension [16]. Many years later in 1992 Asthana et al published a review about the infiltration and stated that while some understanding and control of the process of infiltration has been achieved in recent years, a comprehensive theoretical framework suitable for rationalizing all the observed features of pressure infiltrated systems is lacking [17]. Muscat and Drew studied the infiltration kinetics of molten aluminium into porous titanium carbide. They found that the infiltrated length - time curves had an incubation period prior to stabilizing into theoretically predicted parabolic-type curves. The incubation period became more significant as the infiltration temperature decreased and lasted only after $\sim 50$ seconds. They found that it would be quite impossible to find some mathematical function that can perfectly represent the infiltration due to the complex nature of the influencing parameters [18]. Later Garcia-Cordovilla et al also experienced the incubation in the infiltrated length - time curves and concluded that the origin of the incubation time is not yet fully understood [19]. More recently Kaptay derived theoretical function for infiltrated length and this one is again parabolic in nature and shows linear dependence on the square root of time [20]. In summary it is hard to tell which approximation is the best or at least satisfying. Therefore the best way to get familiar with the behaviour of a system is to measure the kinetics. In order to this and to confirm our preliminary measurements a completely new infiltration equipment was developed as shown in Fig. 5

The system is fully computer controlled in order to avoid any human factor during the measurements and get more reliable 

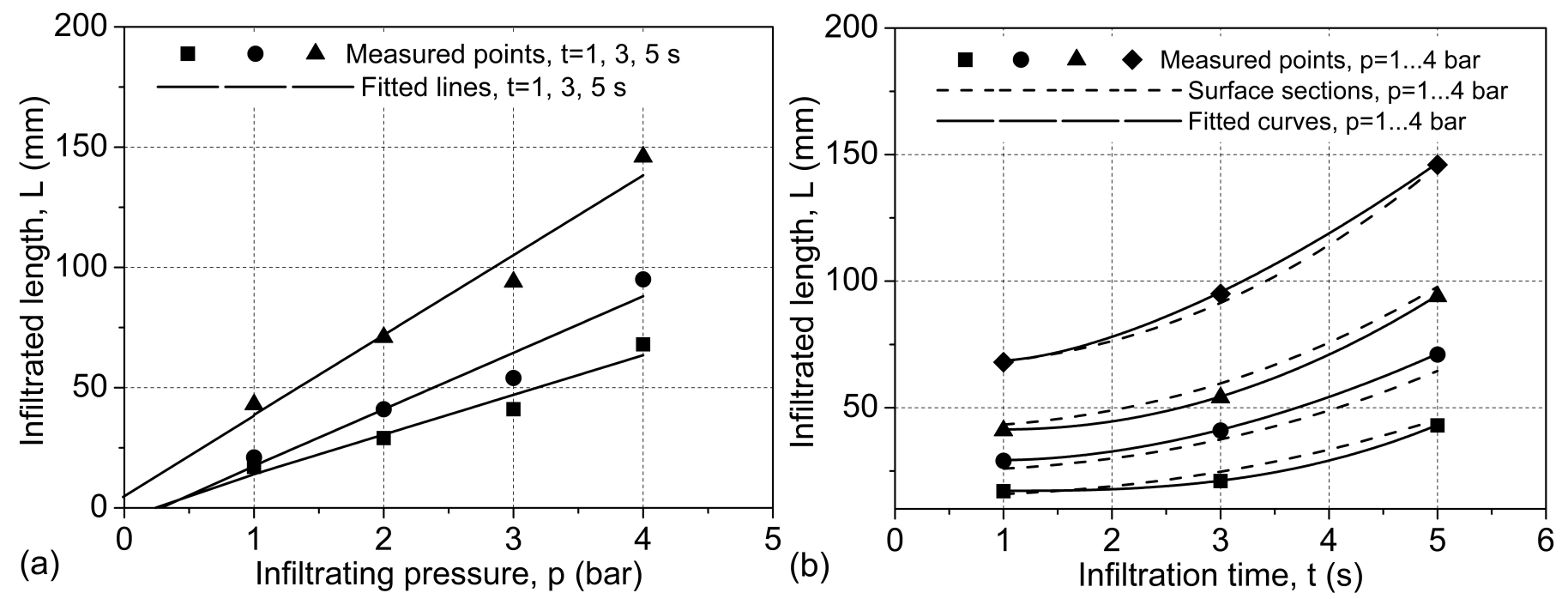

Fig. 5. Measured points, fitted curves and sections of the fitted surfaces: (a) the infiltration time is constant and (b) the infiltration pressure is constant

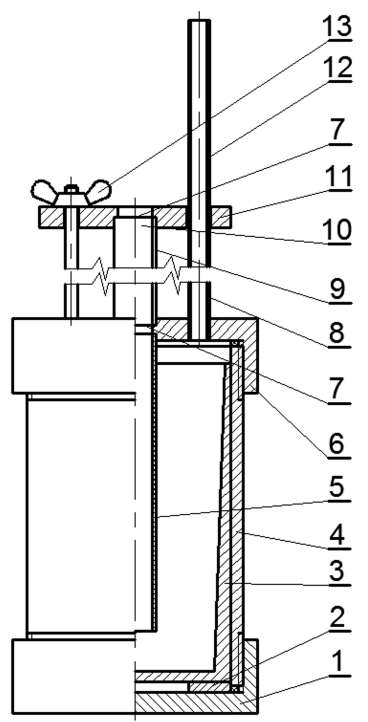

Fig. 6. The advanced measuring system

results. The arrangement of the measuring system can be seen in Fig. 6 .

The principles of the operation is the same, but the whole system is "more aggressive" because three gas inlet (\#8) is applied to ensure faster pressure build up. The microballoon holder pipe (\#9) is also enlarged; it has inner diameter of $16 \mathrm{~mm}$ and length of $200 \mathrm{~mm}$. The system is expanded by a puffer, which has about 50 times larger inner volume, than the equipment itself in order to effective and fast gas inlet. By these modifications the edge effects can be decreased and larger parameter (time and pressure) ranges can be applied. The new measurement system is almost ready to use, nowadays the first tests are running. The new results and their comparison to the preliminary values presented above will be published in a following paper.

\section{Conclusions}

We have found a few theoretical and/or experimental methods to determine threshold pressure for R-CPES infiltration. These

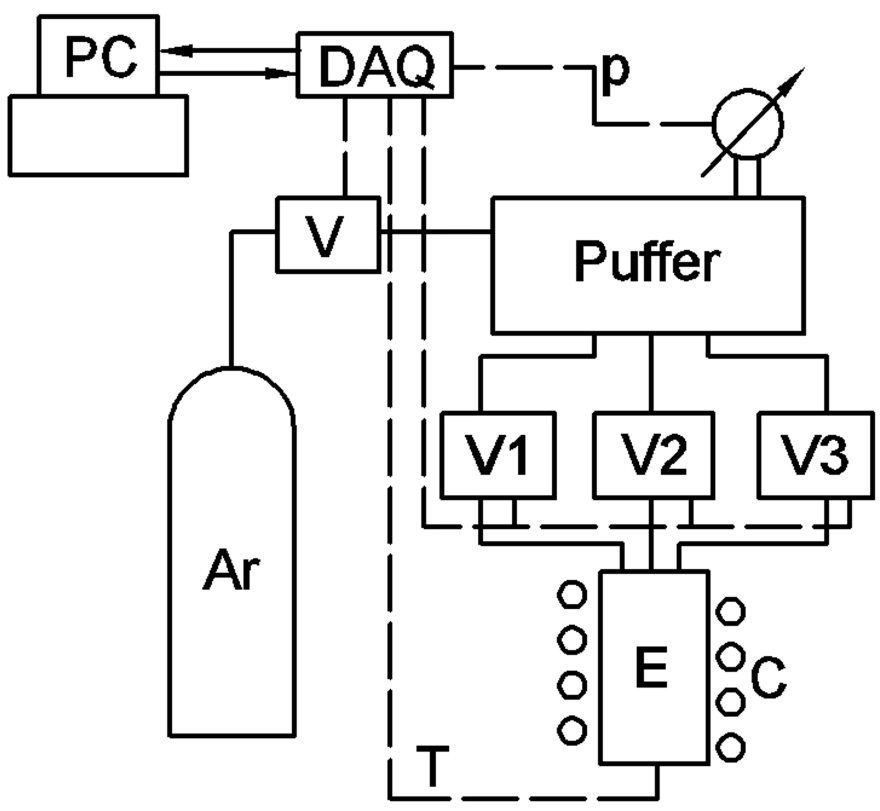

Fig. 7. Arrangement of the new measurements

methods do not consider the effect of time and increased pressure and do not evaluate the infiltrated length. Therefore infiltration equipment was developed, designed and applied to investigate the effects of these parameters on the infiltrated length. The followings can be concluded:

- Power law ruled surface could be fitted on the measured points with good coefficient of determination $\left(\mathrm{R}^{2}\right)$. The calculated fitting parameters ensure the possibility to estimate the infiltrated length for any pressure - time parameters within the measured ranges.

- In the case of fixed time linear fittings were performed. The extrapolated pressures at zero infiltrated length give the threshold pressure, which is between 0.5 and 1 bar for the investigated material pair. For different time parameters the threshold pressures were not the same and this indicates that 
in the case of $t=5$ the wetting conditions were modified by something, maybe the possible chemical reaction between the microballoons and the matrix.

- Our preliminary results do not obey the theoretically predicted square root like curves, the best fitting was given by a power ruled function with the exponent between 1.5 and 2.7 in the measured infiltration time and infiltration pressure ranges. In the professional literature a $\sim 50$ seconds long incubation period of these curves is mentioned and the author believes that this phenomenon was measured within the applied short time range (less than 10 seconds).

- To confirm the results new measurements with extended time and pressure ranges are necessary. Therefore a completely new and advanced infiltration equipment was designed and built with computer control and faster/more reliable operation.

\section{Acknowledgements}

Special thanks to Professor J.T. Blucher for his kind support. The Metal Matrix Composites Laboratory is supported by Grant \# GVOP 3.2.1-2004-04-0145/3.0. This paper was supported by the János Bolyai Research Scholarship of the Hungarian Academy of Sciences. The investigations were supported by The Hungarian Research Fund, NKTH-OTKA PD 83687. This work is connected to the scientific program of the "Development of quality-oriented and harmonized $\mathrm{R}+\mathrm{D}+\mathrm{I}$ strategy and functional model at BME" project. This project is supported by the New Széchenyi Plan (Project ID: TÁMOP-4.2.1/B-09/1/KMR2010-0002). Thanks to R. Tóth and C. H. Erbslöh Hungaria Ltd. for providing E-spheres.

\section{References}

1 Babcsán N, Leitlmeier D, Banhart J, Metal foams-High temperature colloids Part I: Ex situ analysis of metal foams, Colloids and Surfaces A: Physicochemical Engineering Aspects 261 (2005), 123-130, DOI 10.1016/j.colsurfa.2004.12.030.

2 Babcsán N, García Moreno F, Banhart J, Metal foams-High temperature colloids Part II: In situ analysis of metal foams, Colloids and Surfaces A: Physicochemical Engineering Aspects 309 (2007), 254-263, DOI 10.1016/j.colsurfa.2007.02.044.

3 Orbulov I N, Dobránszky J, Producing metal matrix syntactic foams by pressure infiltration, Periodica Polytechnica Mechanical Engineering 52 (2008:1), 35-42, DOI 10.3311/pp.me.2008-1.06.

4 Rohatgi PK, Kim J K, Gupta N, Alaraj S, Daoud A, Compressive characteristics of A356/fly ash cenosphere composites synthesized by pressure infiltration technique, Composites Part A 37 (2006), 430-437, DOI 10.1016/j.compositesa.2005.05.047.

5 Rohatgi P K, Guo R Q, Iksan H, Borchelt E J, Asthana R, Pressure infiltration technique for synthesis of aluminium-fly ash particulate composite, Materials Science and Technology A 244 (1998), 22-30.

6 Palmer R A, Gao K, Doan T M, Green L, Cavallaro G, Pressure infiltrated syntactic foams - Process development and mechanical properties, Materials Science and Engineering A 464 (2007), 85-92, DOI 10.1016/j.msea.2007.01.116.
7 Balch D K, Dunand D C, Load partitioning in aluminum syntactic foams containing ceramic microspheres, Acta Materialia 54 (2006), 1501-1511, DOI 10.1016/j.actamat.2005.11.017.

8 Balch D K, O'Dwyer J G, Davis G R, Cady C M, Gray III G T, Dunand D C, Plasticity and damage in aluminium syntactic foams deformed under dynamic and quasi-static conditions, Materials Science and Engineering A 391 (2005), 408-417, DOI 10.1016/j.msea.2004.09.012.

9 Kiser M, He M Y, Zok F W, The mechanical response of ceramic microballoon reinforced aluminum matrix composites under compressive loading, Acta Materialia 47 (1999:9), 2685-2694.

10 Wu G H, Dou Z Y, Sun D L, Jiang L., Ding B S, He B F, Compression behaviours of cenosphere-pure aluminium syntactic foams, Scripta Materialia 56 (2007), 221-224, DOI 10.1016/j.scriptamat.2006.10.008.

11 Bárczy T, Kaptay Gy., Modeling the infiltration of liquid metals into porous ceramics, Materials Science Forum 297-302 (2005), 473-474.

12 Jaeger H M, Nagel S R, Physics of the granular state, Science 5051 (1992), 1523-1531.

13 Trumble $\mathbf{P}$ K, Spontaneous infiltration of non-cylindrical porosity: closepacked spheres, Acta Materialia 46 (1998), 2363-2367.

14 Envirospheres Ltd (25.05.2011), available at www . envirospheres.com/ products.asp

15 Washburn E W., The dynamics of capillary flow, The Physical Review 1921 17, 273-283.

16 Semlak K A, Rhines F N, The rate of infiltration of metals, Transactions of The Metallurgical Society of AIME 16 (1958), 325-331.

17 Asthana R, Rohatgi P., Tewari N, Infiltration processing of metal-matrix composites: a review, Processing of Advanced Materials 2 (1992), 1-17.

18 Muscat D, Drew R., Modelling the infiltration kinetics of molten aluminum into porous titanium carbide, Metallurgical and Materials Transactions A 25 (1994), 2357-2370.

19 Garcia-Cordovilla C, Louis E, Narciso J, Pressure infiltration of packed ceramic particulates by liquid metals, Acta Materialia 47 (1999:18), 44614479.

20 Kaptay G, Interfacial Aspects to produce particulate reinforced metal matrix composites in Affordable Metal-Matrix Composites for High Performance Applications, Eds.: Pandey AB, Kending KL, Watson TJ. TMS The Minerals Metatals, Materials Society 20 (2001), 72-99. 